\title{
RESÚMENES DE LAS CONFERENCIAS
}

\section{METABOLISMO ÓSEO}

\section{Bloque Simposio - Osteoporosis Diagnóstico de la osteoporosis}

\section{Enrique Ardila}

Médico internista-endocrinólogo

Miembro de número ACE

$\mathrm{E}$ l diagnóstico de la osteoporosis continúa siendo un problema no resuelto. Existen numerosos protocolos, guías, reuniones de consenso etc. que informan sobre cómo tratar esta alteración, pero el diagnóstico sensible y específico continúa sin resolver.

Desde los primeros intentos que tuvieron lugar en los años noventa del siglo pasado y muchos de ellos avalados por las asociaciones científicas internacionales, es poco lo que se ha progresado, muy diferente a lo que ha sucedido con el tratamiento.

Diagnosticar la osteoporosis con base solamente en la densidad ósea que es lo que se utiliza actualmente, es poco incluyente de otros aspectos como la arquitectura ósea y otros que revisten gran importancia como factores de riesgo para la aparición de fracturas. Además de que los parámetros recomendados por la Organización Mundial de la Salud (OMS) fueron llevados a cabo en un grupo poblacional específico y no pueden generalizarse a toda la población.

Los marcadores de recambio óseo tienen aplicabilidad más en el seguimiento que en el diagnóstico y en casos muy específicos.

Posiblemente el paso importante que se ha dado hasta ahora ha sido la creación del FRAX, metodología que ayuda a tener en cuenta otros tipos de factores de riesgo y en poblaciones más específicas.

Esperamos conocer a corto plazo un método sencillo, económico, práctico, sensible y específico que nos permita conocer fácilmente el tipo de paciente que ameritaría tratar.

\section{Bloque Simposio - Salud Masculina} \section{Enfoque diagnóstico y terapéutico de osteosarcopenia en el hombre}

\section{Jorge Alejandro Castillo Barcias}

Médico Internista, Endocrinólogo. Miembro de Número de la Asociación Colombiana de Endocrinología, Diabetes y Metabolismo, Bogotá.

$\mathrm{E}$ término osteosarcopenia no puede ser catalogado como universalmente aceptable, o debe ser incluido como una sola enfermedad, sino debe entenderse como la presencia temporal de dos enfermedades, osteoporosis y sarcopenia, en un mismo paciente.

Sin embargo, desde el punto de vista embriológico, etiológico funcional, y terapéutico, esa "temporalidad" es algo más que un evento casual.

La osteoporosis se define como la presencia de una densidad mineral ósea (DMO), medida por DEXA, en un paciente con un $T$ score menor de $-2,5$.
La sarcopenia no ha terminado de definirse; sin embargo, el consenso europeo la define, con base en tres criterios $^{(1)}$ :

- Baja masa muscular, pacientes con un índice de Masa muscular esquelética (SMI) menor de $7,26 \mathrm{Kg} / \mathrm{m}^{2}$ por DEXA, o menor de $8,87 \mathrm{Kg} / \mathrm{m}^{2}$ por bioimpedanciometría.

- Disminución de la fuerza muscular, una fuerza de empuñadura (handgrip) menor de $30 \mathrm{Kg}$.

- Bajo desempeño físico, dado por una velocidad de la marcha menor de $1 \mathrm{~m} / \mathrm{s}$.

Con la sola disminución de la masa muscular se hace diagnóstico de presarcopenia; si a lo anterior se le agrega alguno de los otros dos criterios, se hace diagnóstico de sarcopenia, con la presencia de los tres criterios, el de sarcopenia severa.

Hay que tener en cuenta que la constitución muscular cambia radicalmente según la etnia, lo que conlleva a la necesidad 
de hacer estudios poblacionales para cada región y a partir de valores de normalidad redefinir la sarcopenia para pacientes con $T$ score menor de -2,0.

El órgano muscular y el esquelético comparten más que una unión estrecha anatómica, enfocada como una unidad funcional de movilidad y que, por lo tanto, el manejo debe ser integral; de hecho, en las recomendaciones del consenso europeo se sugiere evaluar la masa muscular a las pacientes con fracturas asociadas con caídas.

Sabemos que tanto mioblastos como osteoblastos comparten la misma célula mesodérmica multipotencial; el sedentarismo deteriora la masa muscular y el desuso también conduce a la osteoporosis; factores nutricionales como la deficiencia de vitamina D, se asocian a sarcopenia y osteoporosis, y su suplencia es una herramienta eficaz para manejarlas. Factores nutricionales, como el bajo consumo de proteínas, aceleran la pérdida de masa muscular e interfieren con la generación de la matriz ósea. El uso de corticoides afecta por igual al músculo como al esqueleto al igual que el síndrome de Cushing; otros factores hormonales como el hipogonadismo deben descartarse en ambas entidades ${ }^{(2)}$.

El pico de masa muscular y esquelética se presenta alrededor de los 20-30 años, y después de los 50 años se observa en el hombre una pérdida significativa tanto de músculo como de hueso, siendo muy evidente después de los 60 años.

En estudios poblacionales ${ }^{(3)}$, el aumento de una desviación estándar de masa muscular se ha relacionado con una disminución del 37\% del riesgo de osteopenia/osteoporosis; en otros estudios en pacientes en posoperatorio de fractura de cadera, el principal factor predictor de refractura no tuvo ninguna relación con marcadores óseos, sino con variantes asociadas a la sarcopenia, como fuerza muscular y desempeño físico ${ }^{(4)}$.

La prevención de fractura en el hombre debe evolucionar desde una ganancia de masa ósea, hasta la mejoría de la microarquitectura pero también a la ganancia de masa muscular, que se refleje en aumento de la fuerza y desempeño físico; seguir ignorando al músculo es perder la oportunidad de un manejo integral de las fracturas.

\section{Referencias}

1. Alfonso J. Cruz-Jentoft, Jean Pierre Baeyens, Jürgen M. Bauer, Yves Boirie, Tommy Cederholm, et al. Sarcopenia: European consensus on definition and diagnosis. Age and Ageing 2010; 39: 412-423.

2. Camille Tagliaferri, Yohann Wittrant, Marie-Jeanne Davicco, Stéphane Walrand, Véronique Coxam. Muscle and bone, two interconnected tissues. Ageing Research Reviews 21 (2015) 55-70.

3. H. He, Y. Liu, Q. Tian, C. J. Papasian, T. Hu,H.-W. Deng. Relationship of sar- copenia and body composition with osteoporosis. Osteoporos Int (2016) 27:473-482.

4. Bradley D. Lloyd, Dominique A. Williamson, Nalin A. Singh, Ross D. Hansen, Terrence H. Diamond. Recurrent and Injurious Falls in the Year Following Hip Fracture: A Prospective Study of Incidence and Risk Factors From the Sarcopenia and Hip Fracture Study . J Gerontol A Biol Sci Med Sci 2009. Vol. 64A, No. 5, 599-609.

\section{Bloque Simposio - Osteoporosis Terapia osteoformadora en osteoporosis}

\section{Manuel Alberto Pérez Mazorra MD}

Especialista en Medicina Interna y Endocrinología

Miembro de Número Asociación Colombiana de Endocrinología,

Diabetes y Metabolismo

Clínica Central del Quindío - Clínica del Café.

Armenia, Quindio

$\mathrm{L}$ a osteoporosis es un problema de salud común con una prevalencia progresiva tanto en países desarrollados como en vías de desarrollo ${ }^{(1)}$.

De acuerdo con recientes estimaciones de la Federación Internacional de Osteoporosis (IOF) hay 200 millones de mujeres con osteoporosis en el mundo, con un accidente o fractura osteoporótica cada tres segundos ${ }^{(1)}$. Las osteoporosis primaria y secundaria están asociadas con reducción en la masa ósea y la microarquitectura que resulta en fragilidad ósea con un incremento significativo en discapacidad, morbilidad y riesgo de fractura. El acceso al diagnóstico y a una adecuada terapia es un desafío en todo el mundo ${ }^{(2)}$.

Actualmente las medicaciones aprobadas para el tratamiento de la osteoporosis pueden ser divididas en dos grandes categorías, la terapia antirresortiva y la terapia anabólica.

El objetivo es realizar una revisión de la terapia anabólica disponible en la actualidad y las nuevas moléculas que se encuentran en desarrollo.

\section{Teriparatida}

La hormona paratiroidea (PTH) es producida por las glándulas paratiroides y juega un papel principal en la regulación del metabolismo del calcio y el fósforo, una producción continua de PTH puede llevar a consecuencias deletéreas sobre el esqueleto, particularmente sobre el hueso cortical. Sin embargo, la administración intermitente de PTH resulta en un 
incremento en el número y la actividad de los osteoblastos, llevando a un incremento en la masa ósea y a mejoría en la arquitectura esquelética del hueso cortical y trabecular ${ }^{(3,4)}$.

Teriparatida (1-34 PTH recombinante humana) está aprobada como tratamiento inicial de mujeres con osteoporosis posmenopáusica que tienen un riesgo alto de fractura o que han fallado o son intolerantes a previas terapias para osteoporosis. También está aprobada para tratamiento de osteoporosis inducida por glucocorticoides y como terapia para osteoporosis en hombres. La dosis es de 20 mcg una vez por día subcutánea durante dos años ${ }^{(5)}$.

Teriparatida ha mostrado reducir el riesgo de fracturas vertebrales y no vertebrales en mujeres con osteoporosis posmenopáusica. La incidencia de fractura de cadera fue baja en el estudio pero la evidencia sobre protección de fractura de cadera es menor. Teriparatida incrementa dramáticamente la densidad mineral ósea en la columna pero tiene poco efecto sobre la DMO en la cadera o el antebrazo. Pacientes que pierden DMO en la cadera con teriparatida aún están protegidos contra fracturas vertebrales comparados con placebo ${ }^{(6)}$. También tiene evidencia como tratamiento en osteoporosis inducida por esteroides y en osteoporosis en hombres.

Los efectos adversos de teriparatida son leves y transitorios e incluyen náuseas, hipotensión ortostática (la cual usualmente no necesita la suspensión de la medicación, ocurre en asociación con las primeras dosis y responde al asumir posición reclinada) y calambres en piernas. La hipercalcemia, usualmente leve asintomática y transitoria, ha sido observada pero es infrecuente. La hipercalciuria puede ocurrir raramente y puede responder a modificación de la dosis de suplencia de calcio $^{(2)}$.

Teriparatida tiene una advertencia por la ocurrencia de osteosarcomas en ratas tratadas con muy altas dosis (3-50 veces mayor que dosis humana equivalente $)^{(7)}$. Estudios subsecuentes no han mostrado desarrollo de tumores óseos malignos con dosis de teriparatida hasta tres veces mayor a la dosis equivalente en humanos ${ }^{(8,9)}$. No se recomienda en pacientes con riesgo incrementado de osteosarcoma (enfermedad de Paget del hueso, epífisis abiertas, historia de irradiación que incluya el esqueleto o una elevación inexplicada de niveles de fosfatasa alcalina fracción ósea). No debe ser administrado en pacientes con hiperparatiroidismo primario o cualquier forma de secundario no tratado o no resuelto. No está aprobada para uso por un periodo mayor de dos años ${ }^{(2)}$.

\section{PTH recombinante humana 1-84}

Ha sido investigada en el manejo de osteoporosis posmenopáusica. Se ha postulado que la región C terminal de la PTH, la cual falta en la teriparatida, también tiene funciones biológicas en el hueso que son mediadas por receptores específicos para esta región de la hormona.
No existen estudios comparativos entre PTH 1-34 y PTH 1-84, por lo que no se pueden hacer pronunciamientos sobre las potenciales diferencias en efectividad y seguridad ${ }^{(10)}$.

\section{Anticuerpos monoclonales antiesclerostina (romosozumab - blosozumab)}

La esclerostina es un producto del gen Sost que inhibe la osteoblastogénesis. Las mutaciones del gen Sost resultan en expresión ausente de la esclerostina y son responsables de esclerosteosis y de la enfermedad de Van Buchem. Ambas displasias esqueléticas se caracterizan por marcados aumentos de la masa ósea. Los anticuerpos monoclonales humanizados antiesclerostina causan mejoría en la señalización del Wht y aumento de la masa ósea en roedores y primates: en un modelo en ratas posmenopáusicas con osteoporosis el tratamiento con el anticuerpo monoclonal antiesclerostina aumentó la masa ósea e impidió la pérdida ósea asociada con la pérdida de estrógenos ${ }^{(11,12)}$.

En una etapa posterior en humanos, se demostró que los anticuerpos antiesclerostina pueden aumentar la DMO y los marcadores bioquímicos de formación ósea ${ }^{(13,14)}$.

En un estudio de fase 1 con 72 sujetos sanos que recibieron romosozumab dosis única, a los 85 días el medicamento fue bien tolerado y aumentó los marcadores de formación ósea entre $60 \%-100 \%{ }^{(15)}$. En un estudio de fase II, multicéntrico, aleatorizado, controlado con placebo, durante 12 meses se encontró aumento de DMO en columna lumbar, cadera total y cuello femoral en un $11,3 \%, 4,1 \%$ y $3,7 \%$ respectivamente. Igualmente, se encontró disminución en los niveles de telopéptido C (CTXs) ${ }^{(16)}$.

El tratamiento se asoció con disminución dependiente de dosis de los niveles de calcio con un aumento compensatorio de la PTH ${ }^{(16)}$.

Hay varios estudios en fase III que investigan el uso de romosozumab, tanto en mujeres posmenopáusicas como en hombres ${ }^{(16-18)}$.

Se están desarrollando otros anticuerpos antiesclerostina como el blosozumab que se ha investigado en estudios fase I y fase $\mathrm{II}^{(19,20)}$.

\section{Conclusión}

LA teriparatida es una terapia anabólica que mejora la densidad ósea, reduce el riesgo de fractura vertebral y no vertebral. Adicionalmente, hay nuevos fármacos disponibles antiosteoporóticos que estarán disponibles en un futuro próximo, muchos de los nuevos medicamentos combinan eficacia con administración conveniente que podría traducirse en una mejor adherencia. La integración de las terapias disponibles tanto antirresortivas como anabólicas se traducirá en más y mejores opciones para el tratamiento exitoso de la osteoporosis. 


\section{Referencias}

1. Facts and statistics, osteoporosis general. Available at: https://www.iofbonehealth.org/epidemiology Acceso 4 abril 2017.

2. American Association Of Clinical Endocrinologists and American College of Endocrinology. Clinical Practice Guidelines for the Diagnosis and Treatment of Postmenopausal Osteoporosis - 2016.

3. Neuprez A, Reginster JY. Bone-forming agents in the management of osteoporosis. Best Pract Res Clin End Met. 2008;22:869-83.

4. Vahle JL, Sato M, Long GG, Young JK, Francis PC, Engelhardt JA, et al. Skeletal changes in rats given daily subcutaneous injections of recombinant human parathyroid hormone (1-34) for 2 years and relevance to human safety. Toxicol Pathol. 2002;30:312-321.

5. Neer RM, Arnaud CD, Zanchetta JR, Prince R, Gaich GA, Reginster JY, et al. Effect of parathyroid hormone (1-34) on fractures and bone mineral density in postmenopausal women with osteoporosis. N Engl J Med. 2001; 344:1434-1441.

6. Watts NB, Miller PD, Kohlmeier LA, Sebba A, Chen P, Wong M, et al. Vertebral fracture risk is reduced in women who lose femoral neck BMD with teriparatide treatment. J Bone Miner Res. 2009;24:1125-1131.

7. Vahle JL, Long GG, Sandusky G, Westmore M, Ma YL, Sato M. Bone neoplasms in F344 rats given teriparatide [rhPTH(1-34)] are dependent on duration of treatment and dose. Toxicol Pathol. 2004;32:426-438.

8. Harper KD, Krege JH, Marcus R, Mitlak BH. Osteosarcoma and teriparatide? J Bone Miner Res. 2007; 22:334.

9. Subbiah V, Madsen VS, Raymond AK, Benjamin RS, Ludwig JA. Of mice and men: divergent risks of teriparatide-induced osteosarcoma. Osteoporos Int. 2010;21:1041-1045.

10. Verhaar HJ, Lems WF. PTH-analog: comparable or different? Arch Gerontol Geriatr. 2009;49(2):130-2.

11. Canalis E. Update in new anabolic therapies for osteoporosis. J Clin Endocrinol Metab. 2010;95(4):1496-504.
12. Watts NB, Bilezikian JP. Advances in target-specific therapy for osteoporosis. J Clin Endocrinol Metab. 2014;99(4):1149-51.

13. Weivoda MM, Oursler MJ. Developments in sclerostin biology: regulation of gene expression, mechanisms of action, and physiological functions. Curr Osteoporos Rep. 2014;12(1):107-14.

14. Lewiecki EM. Sclerostin monoclonal antibody therapy with AMG 785: a potential treatment for osteoporosis. Expert Opin Biol Ther. 2011;11(1):11727.

15. Padhi D, Jang G, Stouch B, Fang L, Posvar E. Single-dose, placebo-controlled randomized study of AMG 785, a sclerostin monoclonal antibody. J Bone Miner Res. 2011;26(1):19-26.

16. Padhi D, Allison M, Kivitz AJ, Gutiérrez MJ, Stouch B, Wang C, et al. Multiple doses of sclerostin antibody romosozumab in healthy men and posmenopausal women with low bone mass: a randomized, double-blind, placebocontrolled study. J Clin Pharmacol. 2014;54(2):168-78.

17. McClung MR, Grauer A, Boonen S, Bolognese MA, Brown JP, Diez-Perez A, et al. Romosozumab in postmenopausal women with low bone mineral density. N Engl J Med. 2014;370(5):412-20.

18. Becker CB. Sclerostin inhibition for osteoporosis-a new approach. N Engl J. Med. 2014;370(5):476-7.

19. McColm J, Hu L, Womack T, Tang CC, Chiang AY. Single and multipledose randomized studies of blosozumab, a monoclonal antibody against sclerostin, in healthy postmenopausal women. J Bone Miner Res. 2014 Apr;29(4):935-43.

20. Recker R, Benson C, Matsumoto T, Bolognese MA, Robins DA, Alam J, et al. A randomized, double-blind phase 2 clinical trial of blosozumab, a sclerostin antibody, in postmenopausal women with low bone mineral density. J Bone Miner Res. 2015;30(2):216-24.

\section{Precongreso - Osteoporosis, Metabolismo del Calcio y de la Vitamina D Metabolismo de la vitamina D}

\section{Amanda Páez Talero}

Médica Endocrinóloga. Miembro de Número de la Asociación

Colombiana de Endocrinología, Diabetes y Metabolismo, Bogotá.

$\mathrm{L}$ a vitamina D es fundamental en el metabolismo óseo, su forma activa, la 1,25 dihidroxivitamina D, 1,25 $(\mathrm{OH}) 2 \mathrm{D}$ o calcitriol, se une a un receptor hormonal específico ( VDR) para ejercer todas sus funciones, tanto a nivel óseo como en otros tejidos. En el caso del tejido óseo su deficiencia produce alteraciones del metabolismo fosfocálcico, hiperparatiroidismo secundario, pérdida de masa ósea e incremento del riesgo de fractura, la deficiencia extrema produce osteomalacia.

En cuanto a los efectos de la 1,25 (OH)2D sobre otros sistemas es conocida la presencia de receptores VDR en células de piel, músculo estriado y liso, promielocitos, linfocitos, colon, hipófisis, ovarios y células endoteliales. Numerosos estudios han buscado aclarar el hallazgo de hipovitaminosis D en desenlaces como sarcopenia, aumento del riesgo de caídas, alteraciones del sistema inmune con incremento de enfermedades bacterianas, cáncer de seno próstata y colon; así como la asociación con obesidad, síndrome metabólico, ovario poliquístico y aumento del riesgo cardiovascular.

La vitamina D3 o colecalciferol se sintetiza a partir del 7-dehidrocolesterol en la piel de los animales superiores por el efecto sobre ésta de los rayos ultravioleta, otro tanto ocurre en los vegetales para la generación de vitamina D2 o ergocalciferol; posterior a esta síntesis, la vitamina D3 sale a la circulación y a su paso por el hígado sufre una primera hidroxilación en la posición 25 produciendo la $25(\mathrm{OH})$ VD o calcidiol que se une a una proteína ligadora de vitamina $\mathrm{D}$ y a otras provenientes de la superfamilia de albúminas, la 25(OH)VD es la forma más abundante de la vitamina D y la que resulta ideal para su determinación en el laboratorio. Esta prohormona a su paso a través del riñón sufre una segunda hidroxilación por una alfa hidroxilasa mitocondrial la CYP27B1, que al ubicarse en la posición 1 alfa le confiere a la 1-alfa, $25(\mathrm{OH}) 2 \mathrm{VD}$ o calcitriol una alta afinidad para unirse a su receptor VDR en los tejidos blanco. La 1,25 (OH)2D circula en el suero a concentraciones del $0,1 \%$ de su prohormona la $25(\mathrm{OH}) \mathrm{VD}$.

La vitamina D, llamada por algunos autores "hormona D" por sus características de acción a través de receptores nucleares, regula funciones endocrinas, autocrinas y paracrinas.

La fuente principal de la hidroxilasa CYP27B1 está en el túbulo proximal del riñón y la formación de1,25 (OH)2D a este nivel está estimulada por la PTH, estrógenos y la hipofosfatemia y es inhibida por el factor de crecimiento de fibroblastos 23 (PGF23) producido en los osteocitos, la calcitonina y por sí misma. De esta forma, cuando los niveles de 25(OH)VD están bajos, el aumento de PTH circulante normaliza los niveles de 
1,25 (OH)2D, esta vitamina se almacena habitualmente en hígado, músculo y tejido graso.

La función más evidente de la 1,25 $(\mathrm{OH}) 2 \mathrm{D}$ es el mantenimiento de la normocalcemia mediante su actuación a nivel intestinal, óseo, renal y en la paratiroides.

A nivel intestinal la 1,25 $(\mathrm{OH}) 2 \mathrm{D}$ se une al receptor VDR, regulando el ADN nuclear y la transcripción del ARN mensajero, controlando la síntesis de la fosfatasa alcalina, el colágeno, la calbindina; para promover la absorción de calcio por difusión facilitada. Las otras proteínas facilitan la proliferación y diferenciación en las microvellosidades. La vía no genómica regula el transporte rápido de calcio, llamado transcaltaquia, que consiste en la apertura de canales de calcio operados por cambios de voltaje a través de la membrana.

Las acciones óseas se dan por diferentes mecanismos, la vitamina D aumenta el número y la función de los osteoblastos quienes expresan la 1 alfa hidroxilasa y la 24 hidroxilasa, al activarse por la unión al VDR permiten la síntesis de osteocalcina y la formación ósea. También modulan los osteoclastos; en el déficit de 1,25 (OH)2D, esta modulación osteoclástica se pierde y se incrementa la síntesis de RANKL en osteoblastos y en condrocitos, aumentando el cociente RANKL/osteoprotegerina, siendo este el principal mecanismo responsable de la activación de la actividad ósea resortiva.

Clásicamente se acepta que la deficiencia de vitamina D impide la mineralización del osteoide, causando una severa enfermedad ósea que denominamos osteomalacia.

El mecanismo renal de regulación de la vitamina D ya se enunció arriba, el túbulo renal es la fuente principal de la hidroxilasa CYP27B1, precursora de la 1,25 (OH)2D, la regulación de este sustrato está mediada por PTH e inhibida por el PGF23 producido en los osteocitos.

\section{Impacto de la vitamina $D$ en otros tejidos}

Una función primaria de la 1,25 (OH)2D es la protección interna del huésped, ésta se produce como una citocina en monocitos y macrófagos y actúa en forma intracrina por interacción con el VDR para modular la respuesta inmune contra agentes microbianos, al producirse en suficiente cantidad puede expresarse por vía intracrina a través del VDR de linfocitos $\mathrm{T}$ y B, produciendo respuestas inflamatorias locales. Una diferencia importante entre el CYP27B1 renal y el extrarrenal es que ante niveles bajos del sustrato de 25(OH)VD, la PTH aumentada logra incrementar la 1,25 $(\mathrm{OH}) 2 \mathrm{D}$ renal pero no la extrarrenal, comprometiendo las acciones intracrinas de la hormona en los otros sistemas.

Recientemente se han podido establecer unos patrones de activación generados por la membrana de la bacteria que inician la expresión de la hidroxilasa CYP27B1 en el macrófago; en presencia de bajo nivel de 1,25 (OH)2D no es posible realizar la activación intracrina, activar al VDR y generar cathelicidin (defensina) sustancia que actúa como destructora del microbio.

Finalmente, se evidencia una correlación inversa entre los niveles de 25(OH)VD y la enfermedad cardiovascular, el síndrome metabólico, hipertensión, obesidad e intolerancia a la glucosa. Los mecanismos posibles son:

1. El aumento del tejido graso atrape la $25(\mathrm{OH}) \mathrm{VD}$ y a este nivel la leptina producida por este tejido graso inactive el ciclo local de hidroxilación por la CYP27B1 a 1,25 $(\mathrm{OH}) 2 \mathrm{D}$, limitando la acción de la hormona.

2. Hay receptores VDR en la célula endotelial y en el músculo liso de las arterias

3. La 1,25 (OH)2D tiene un efecto modular del sistema renina-angiotensina, siendo regulador negativo de la renina y de la tensión arterial.

4. El calcitriol afecta el metabolismo lipídico, disminuyendo la apolipoproteína A-1.

\section{Referencias}

1. Adams JS and Hewison M. Update in Vitamin D. 2010 J Clin Endocrinol Metab 95:471-478.

2. Sanchez A. Vitamina D: Actualización. 2010 Rev. Med: Rosario. 76:0-87

\section{Bloque Simposio - Osteoporosis Bisfosfonatos ¿cuándo y hasta cuándo?}

\section{Doly Nubia Pantoja Guerrero}

Médica Endocrinóloga. Miembro de Número de la Asociación Colombiana de Endocrinología, Diabetes y Metabolismo, Bogotá.

$\mathrm{L}$ a osteoporosis es una enfermedad metabólica crónica caracterizada por incremento en el recambio óseo, progresiva pérdida de masa ósea, deterioro de la microarquitectura e incremento en el riesgo de fractura ${ }^{(1)}$. Las indicaciones actuales de tratamiento por la Fundación Internacional de Osteoporosis (IOF) ${ }^{(2)}$ son: pacientes con fractura osteopo- rótica previa, pacientes con una densidad mineral ósea (DMO) T score $<-2,5$ y pacientes mayores de 50 años quienes tienen baja densidad mineral ósea (T score $-1,0$ a $-2,5$ ) y alto riesgo de fractura por FRAX ${ }^{(2)}$.

Los bifosfonatos (BP) son los medicamentos más comúnmente usados en tratamiento en osteoporosis ${ }^{(3)}$. Estos se unen a la hidroxiapatita ósea, alterando la habilidad de resorción de los osteoclastos, induciendo apoptosis de estos e incrementando la DMO con reducción del riesgo de fractura entre 
50\%-70\% ${ }^{(4)}$, los BP se acumulan en el hueso, su efecto inhibitorio sobre los osteoclastos puede durar años después de la suspensión de su uso ${ }^{(5)}$ este mecanismo ha llevado a mucha controversia en el tiempo de uso de los mismos y no se conoce con exactitud por cuánto tiempo después de la suspensión el paciente todavía está protegido, a pesar de tratamiento de DMO a nivel de cadera adicional a ello con los reportes iniciales de Osteonecrosis de mandíbula en 2003 y de Fracturas Femorales Atípicas (FFA) y su probable relación con uso de $\mathrm{BP}^{(6)}$ llevó a la FDA a revisar en 2011 todos los datos de seguridad y eficacia de los BP y recomendó a los médicos revaluar la indicación para continuación de la terapia más allá de 3-5 años ${ }^{(7-8)}$. Al momento se habla de drug Holiday o suspensión transitoria del medicamento de acuerdo con el nivel de riesgo de fractura del paciente.

La evidencia actual que soporta el uso de BP a largo plazo más allá de cinco años es derivada de dos estudios aleatorizados, doble ciego que fueron realizados en Estados Unidos y Europa con alendronato (Estudio FLEX) y con ácido zoledrónico (Estudio de Extensión HORIZON) ${ }^{(9-10)}$. El estudio FLEX fue un estudio de extensión del estudio FIT (alendronato Fracture Intervention Trial) que aleatorizó 1.099 mujeres posmenopáusicas quienes recibieron alendronato durante 4 o 5 años y fueron aletorizadas a continuar con placebo o alendronato, encontrando menor incidencia de fracturas vertebrales clínicas 5,3\% vs. $2,4 \%$ en el grupo que continuó con alendronato $(R R=0,45$, IC 95\% 0,24, 0,85), no se encontró diferencia en las fracturas vertebrales morfométricas ni diferencia en las fracturas no vertebrales entre las pacientes que continuaron con alendronato vs. las pacientes que suspendieron el tratamiento ${ }^{(9-10)}$. En el estudio de extensión HORIZON, 1.233 mujeres posmenopáusicas que habían recibido tres infusiones anuales de $5 \mathrm{mg}$ de ácido zoledrónico (ZOL) fueron aleatorizadas a continuar con ZOL durante tres años adicionales o con placebo, encontrando menor incidencia de fracturas vertebrales morfométricas en un $52 \%$ en el grupo que continuó con tratamiento a seis años ${ }^{(11-12)}$ no existió diferencia en las fracturas vertebrales clínicas ni en las fracturas no vertebrales ${ }^{(11-12)}$
Con la idea de identificar subgrupos de pacientes que se beneficien con los BP a largo plazo, en los dos estudios mencionados previamente los investigadores realizaron análisis post-hoc para identificar los grupos de mayor riesgo, concluyendo que uno de los marcadores para clasificar a un paciente en alto riesgo es la persistencia a pesar de tratamiento de DMO a nivel de cadera de acuerdo al estudio FLEX de -2 y $-2,5$ y al HORIZON $<2,5$.

El reporte del TaskForce de la Sociedad Americana para la Investigación Ósea y Mineral (ASBMR ) ${ }^{(13)}$ publicado en enero de 2016, determina que se debe revalorar la continuación de BP de la siguiente manera: para BP orales a los cinco años y para ZOL a los tres años. Para considerar continuar tratamiento se debe tener en cuenta: antecedente de fractura de cadera, columna o múltiples fracturas osteoporóticas previas al inicio de la terapia, presencia de fractura osteoporótica mayor (columna, cadera, húmero o antebrazo), persistencia de $T$ score $<2,5$ o alto riesgo de fractura (ejemplo uso de glucocorticoides). Se debe considerar continuar la terapia oral hasta por diez años y ZOL durante seis años. En los pacientes que tienen una fractura osteoporótica durante el tratamiento debe considerarse la búsqueda de causas secundarias, nuevos factores de riesgo y valoración de adherencia. Adicionalmente, el cambio a terapias alternativas puede ser considerado aunque no se han realizado estudios adecuados para evaluar la eficacia de este enfoque ${ }^{(13)}$.

En cuanto a la suspensión de BP y el reinicio de tratamiento es aún más incierto, por el momento contamos con la opinión de expertos, quienes consideran que podría ser razonable no reiniciar tratamiento si la densidad mineral ósea permanece estable y reiniciar tratamiento con BP (o una terapia alternativa para osteoporosis) si la DMO T-score es $\leq 2,5$, o hay nuevos factores de riesgo ${ }^{(1-13)}$.

El riesgo de osteonecrosis de la mandíbula y FFA aumenta con la duración de la terapia de BP, pero estos eventos son raros contrarrestados con la reducción del riesgo de fractura con $\mathrm{BP}$ en pacientes de alto riesgo. Para las mujeres que no tienen alto riesgo de fracturas después de 3-5 años de tratamiento con BP, se puede considerar la suspensión del tratamiento con revaluación periódica cada dos o tres años ${ }^{(13)}$.

\section{Referencias}

1. Jordan C. Villa, MD \& Arianna Gianakos, BS \& Joseph M. Lane, MD. Bisphosphonate Treatment in Osteoporosis: Optimal Duration of Therapy and the Incorporation of a Drug Holiday. HSSJ (2016) 12:66-73.

2. Moeney D. Briefing information for the September 9, 2011 joint meeting of the advisory. Committee for Reproductive Health Drugs and the Drug Safety and Risk. Management AdvisoryCommittee. 2011;Appendix 8.

3. FDA. [[Accessed May 1, 2014]] Background Document for Meeting of Advisory Committee 2011.

4. Russell RG. Bisphosphonates: the first 40 years. Bone. 2011; 49(1):2-19. [PubMed: 21555003].

5. Lin JH. Bisphosphonates: a review of their pharmacokinetic properties. Bone. 1996; 18(2): 75-85.

6. Schilcher J, Koeppen V, Aspenberg P, Michaëlsson K. Risk of atypical femoral fracture during and after bisphosphonate use. N Engl J Med. 2014; 371(10):974-976. [PubMed: 25184886].

7. Whitaker M, Guo J, Kehoe T, Benson G. Bisphosphonates for osteoporosiswhere do we go from here? N Engl J Med. 2012; 366(22):2048-2051. [PubMed: 22571168].

8. Whitaker M, Guo J, Kehoe T, Benson G. Bisphosphonates for osteoporosiswhere do we go from here? N Engl J Med. 2012; 366(22):2048-2051. [PubMed: 22571168].

9. Black DM, Schwartz AV, Ensrud KE, et al. Effects of continuing or stopping alendronate after 5 years of treatment: the fracture intervention trial longterm extension (FLEX): a randomized trial. JAMA. 2006; 296(24): 2927-2938.

10. Bone HG, Hosking D, Devogelaer JP, et al. Ten years' experience with alendronate for osteoporosis in postmenopausal women. N Engl J Med. 2004; 350(12):1189-1199.

11. Black DM, Delmas PD, Eastell R, et al. Once-yearly zoledronic acid for treatmen of postmenopausal osteoporosis. N Engl J Med. 2007; 356(18): 1809-1822.

12. Black DM, Reid IR, Boonen S, et al. The effect of 3 versus 6 years of zoledronic acid treatment of osteoporosis: a randomized extension to the HORIZONpivotal fracture trial (PFT). J Bone Miner Res. 2012;27(2):243-254.

13. Robert A. Adler, Ghada El-HajjFuleihan, Douglas C. Bauer, Pauline M. Camacho, Bart L. Clarke, Gregory, et al . ASBMR TaskForceonManaging Osteoporosis Patientsafter Long - TermBisphosphonateTreatment. J BoneMiner Res. 2016; 31 (1): 16-35. [PubMed: 22836222]. 